\title{
ANALYZING ANOMALOUS ARTEFACTS IN TDS-1 DELAY DOPPLER MAPS
}

\author{
Changjiang Hu, ${ }^{* 1}$ Craig Benson, ${ }^{1}$ Hyuk Park, ${ }^{2}$ Adriano Camps $^{3}$ Li Qiao, ${ }^{1}$ and Chris Rizos ${ }^{4}$ \\ ${ }^{1}$ School of Engineering and Information Technology, UNSW, Canberra, Australia \\ ${ }^{2}$ Department of Physics, Universitat Politecnica de Catalunya, Spain \\ ${ }^{3}$ Department of Signal Theory and Communications, Universitat Politecnica de Catalunya and \\ IEEC/UPC, Spain \\ ${ }^{4}$ School of Civil and Environmental Engineering, UNSW, Sydney, Australia \\ *Corresponding author: changjiang.hu@student.adfa.edu.au
}

\begin{abstract}
Global Navigation Satellite System Reflectometry (GNSSR) uses the GNSS reflected signals to study parameters of the Earth's surface such as ocean surface height, wind speed, soil moisture, sea surface target detection. In this paper fourteen DDMs (Delay Doppler Maps) of TechDemoSat-1 (TDS-1) containing anomalous artefacts are presented and analyzed. Anomalous artefacts are not caused by the reflection from Earth surface targets, occultation, nor the leakages of direct signals, but likely according to their delays- from reflection of targets above the Earth's surface (either airborne or spaceborne).
\end{abstract}

Index Terms - GNSS, GNSS-R, DDMs

\section{INTRODUCTION}

The use of reflected global navigation signals for Earth observation (known today as GNSS-R) was first proposed in 1988 [1]. Since then a number of applications have been proposed and evaluated. It is found that GNSS-R can be applied to study a range of parameters of the Earth's surface, including ocean surface height [2], wind speed [3], soil moisture [4, 5], snow depth [6], ice detection [7], sea surface target detection [8], oil spill mapping [9], and ionospheric monitoring [10]. In recent years space-based GNSS-R, where receivers are fixed on LEO (Low Earth Orbit) satellites, has attracted greater attention in the GNSS community. Several missions carrying onboard GNSS-R instruments have been launched or are in preparation [1118]. TDS- 1 and CYGNSS are two missions are providing data for GNSS-R research.

This paper found that some TDS-1 DDMs contain anomalous artefacts which are different from normal DDMs. Possible reasons are considered in this paper. Results suggest that the anomalous artefacts are the reflections from targets not on the Earth's surface, but in the air or in space. This study suggests a new application of the GNSS-R; i.e. target detection above the Earth's surface, when the geometry is suitable.

\section{DATA INTRODUCTION}

This paper uses fourteen DDMs of TDS- 1 collected on 14 and 15 September 2018, as shown in Fig. 1. Each DDM is referred to as an event. The location of the specular point of each event is shown in Fig. 2, which is marked by a red star. It can be seen from Fig. 2 that the reflecting bodies of the fourteen events include water and land. Water reflection usually produces a clear "horseshoe" pattern in DDMs, such as the DDMs of Events 11 and 14 of Fig. 1; whereas land reflections usually do not exhibit such clear "horseshoe" pattern (i.e. coherent scattering dominates), such as the DDMs of Events 5 and 13 of Fig. 1. The anomalous artefacts discussed in this paper are the bright points in the red circles of Fig. 1. Table I lists the main information concerning the fourteen DDMs, which is used in the following analyses. More details about TDS-1 data product can be found in [19].

\section{METHODOLOGY}

For normal DDMs, the bright points of Fig. 1 are unexpected. There are four possible causes for the bright points: 1) reflection from an Earth's surface target, 2) GNSS radio-occultation, 3) leakage of GNSS direct signals, or 4) reflection from targets above the Earth's surface. The following analyses discuss the first three causes and conclude that they are unlikely.

\subsection{Reflection from an Earth's surface target}

It can be seen from Fig. 1 that the bright points are located in delay bins before the "horseshoe" pattern, i.e. before the specular reflection from the Earth's surface arrives. These regions are often called the "forbidden zone" of the DDM because they correspond to delays shorter than the delay associated with the points over the Earth's surface. Therefore the bright points of Fig. 1 are not caused by reflections from an Earth surface target. 


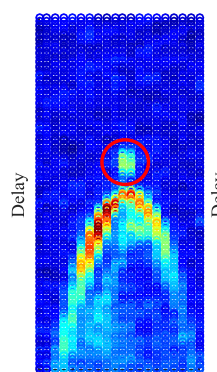

Doppler

Event 1

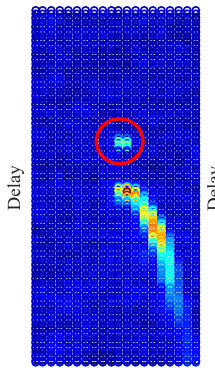

Doppler

Eventer 8

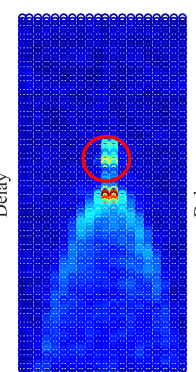

Doppler

Event 2

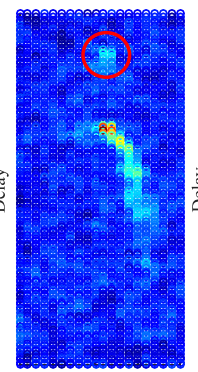

Doppler

Event 9

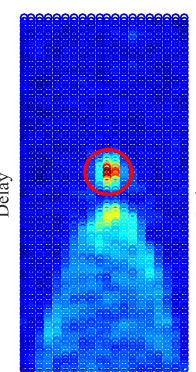

Doppler

Event 3

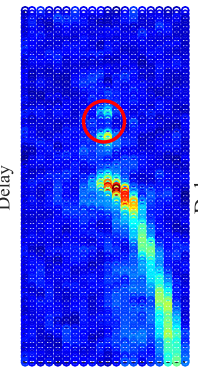

Doppler

Event 10

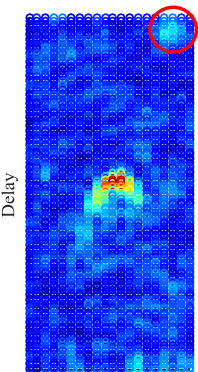

Doppler

Event 4

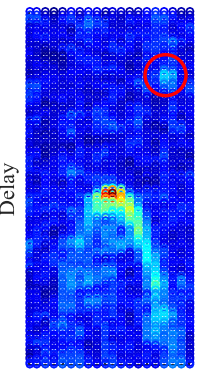

Doppler

Event 11

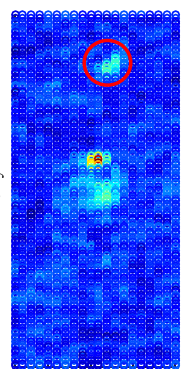

Doppler

Event 5

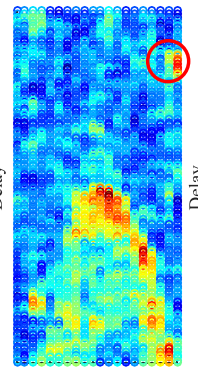

Doppler

Event 12

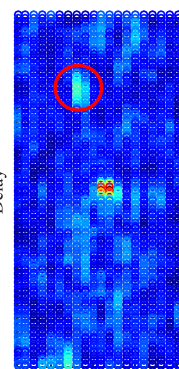

Doppler

Event 6

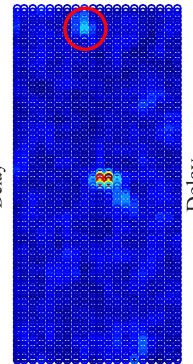

Doppler

Event 13

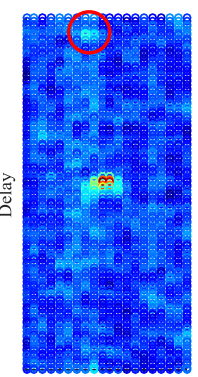

Doppler

Event 7

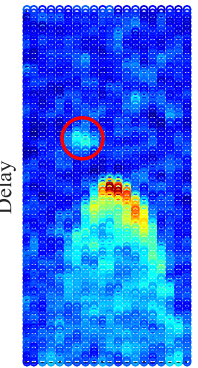

Doppler

Event 14

Fig.1. Sample fourteen DDMs of TDS-1 containing anomalous artefacts (marked by red circles).

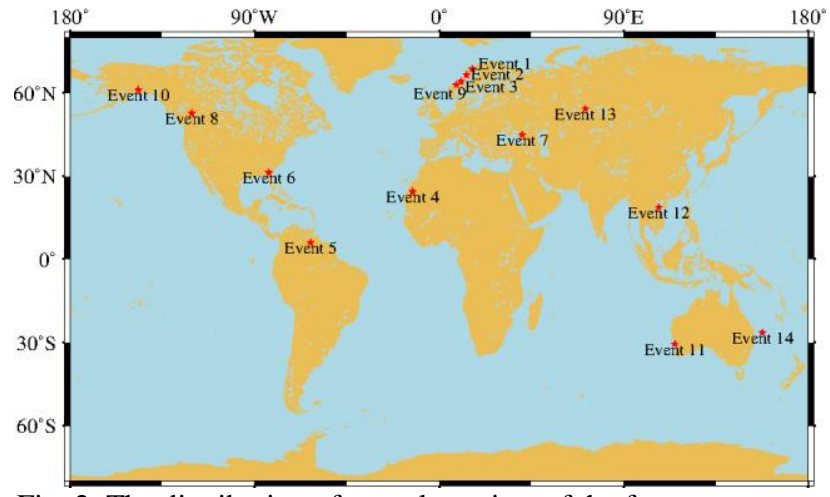

Fig. 2. The distribution of specular points of the fourteen events.

\subsection{Occultation}

The elevation angles of the fourteen events are listed in Table I. It can be seen that the elevation angles are greater $59^{\circ}$, which precludes radio-occultation from happening.

\subsection{Leakage of direct signal}

The upper delay window of the DDM of TDS- 1 is defined as:

$$
L_{s p}-L_{x}<D p \cdot D r \cdot c \cdot \eta
$$

where $L_{s p}$ is the reflected path length through the specular point, $L_{x}$ is the reflected path length via any point, $D p$ is the number of delay pixels (128 for TDS-1 DDMs), $D r$ is the delay resolution of DDM (244 ns), $c$ is the speed of light, and $\eta$ is the ratio between the number of pixels above the specular point and the number of delay pixels. The upper delay window is the necessary and insufficient condition
Table I. Information of the fourteen DDMs.

\begin{tabular}{|c|c|c|c|}
\hline & $\begin{array}{c}\text { Elevation } \\
\text { angle }\end{array}$ & $\begin{array}{c}\text { Column No. } \\
\text { of bright point }\end{array}$ & $\begin{array}{c}\text { Direct Signal } \\
\text { In DDM }\end{array}$ \\
\hline Event 1 & $71^{\circ}$ & 11 & False \\
\hline Event 2 & $72^{\circ}$ & 11 & False \\
\hline Event 3 & $73^{\circ}$ & 11 & False \\
\hline Event 4 & $64^{\circ}$ & 17 & False \\
\hline Event 5 & $85^{\circ}$ & 12 & False \\
\hline Event 6 & $59^{\circ}$ & 8 & False \\
\hline Event 7 & $67^{\circ}$ & 8 & False \\
\hline Event 8 & $77^{\circ}$ & 11 & False \\
\hline Event 9 & $76^{\circ}$ & 11 & False \\
\hline Event 10 & $67^{\circ}$ & 11 & False \\
\hline Event 11 & $61^{\circ}$ & 17 & False \\
\hline Event 12 & $66^{\circ}$ & 20 & False \\
\hline Event 13 & $73^{\circ}$ & 9 & False \\
\hline Event 14 & $59^{\circ}$ & 8 & False \\
\hline
\end{tabular}

that a reflected signal is above the "horseshoe". Given that the specular point is basically located in the mid delay axis of the DDMs, $\eta$ is set to 0.5 in this paper. Therefore the upper delay window is less than $4682 \mathrm{~m}$, e.g. $L_{s p}-L_{x}<$ $4682 m$

Delay and Doppler are analyzed first to decide if the bright points in Fig. 1 are due to leakages of direct signals. The delay and Doppler are simulated using the TDS-1 orbit, precise orbit of transmitter, WGS-84 model, corrections of ionosphere and troposphere delay, and correction of mean sea surface height by the DTU13 model. Two parameters, $\rho_{\text {rem }}$ for the delay and $D_{d}^{S}$ for the Doppler frequency, are obtained from the simulations:

$$
\rho_{\text {rem }}=L_{s p}-L_{d}-k \cdot \tau \cdot c
$$




$$
D_{d}^{s}=D_{\text {dir }}^{s}-D_{\text {ref }}^{s}
$$

where $L_{d}$ is the path length of the direct signal, $\tau$ is the time period of the CA code (1ms), $k$ is an integer ambiguity to make sure $\rho_{\text {rem }}$ is between 0 and $\tau \cdot c \approx 300 \mathrm{~km}, D_{\text {dir }}$ and $D_{\text {ref }}$ are the simulated Doppler of the direct and reflected signals, respectively. In addition, the difference between the observed direct and reflected Doppler $D_{d}^{o}$ can be obtained from DDMs [19]:

$$
D_{d}^{o}=(N-11) \cdot \text { Dre }
$$

where $N$ is the column number of the bright point, and Dre is the Doppler resolution $(500 \mathrm{~Hz}$ in TDS-1). The column numbers of the bright points are listed in Table I. The specular reflection (DDM peak) are set to be in the $11^{\text {th }}$ column in the TDS-1 DDM. A small difference between $D_{d}^{S}$ and $D_{d}^{o}$ is a necessary condition if the anomalous signals were leakages of direct signals.

The value of $\rho_{\text {rem }}$ is used to decide whether the direct signal could be above the "horseshoe" of DDMs. In the case of the direct signal leakage, the delay difference between the two signals is less than $4682 \mathrm{~m}$ (considering the repetition of the code). Therefore, $\rho_{\text {rem }}$ should be less than $4682 \mathrm{~m}$.

Table II lists the values of $\rho_{\text {rem }}, D_{\text {ref }}^{S}, D_{d}^{S}$ and $D_{d}^{o}$ of the fourteen DDMs. It can be seen that the $\rho_{\text {rem }}$ of DDMs range from $15.1 \mathrm{~km}$ to $292.6 \mathrm{~km}$, which are considerably outside the upper delay window. Therefore direct signals cannot be above the "horseshoe" of the DDMs, despite the fact that some DDMs have small difference between $D_{d}^{s}$ and $D_{d}^{o}$, such as Events 3,7 and 13. Thus the bright points in Fig. 1 cannot be due to leakages of direct signals.

In the $\mathrm{L} 1 \mathrm{~b}$ data product there is a system parameter called "DirectSignalInDDM" which indicates if a DDM contains direct signals [19]. Table I shows that the "DirectSignalInDDM" of the fourteen DDMs are "False", which indicates that the DDMs do not contain direct signals.

Table II. The values of $\rho_{\text {rem }}, D_{\text {ref }}^{S}, D_{d}^{S}$, and $D_{d}^{o}$ for the fourteen DDMs.

\begin{tabular}{|c|c|c|c|c|}
\hline & $\begin{array}{c}\rho_{\text {rem }} \\
(\mathrm{km})\end{array}$ & $\begin{array}{c}D_{\text {ref }}^{s} \\
(\mathrm{~Hz})\end{array}$ & $D_{d}^{s}(\mathrm{~Hz})$ & $D_{d}^{o}(\mathrm{~Hz})$ \\
\hline Event 1 & 273.1 & 6703 & 1701 & 0 \\
\hline Event 2 & 284.4 & 4726 & 1177 & 0 \\
\hline Event 3 & 292.6 & 2242 & 518 & 0 \\
\hline Event 4 & 186.7 & 2081 & 453 & 3000 \\
\hline Event 5 & 54.7 & -3114 & -745 & 500 \\
\hline Event 6 & 116.3 & 15812 & 3826 & -1500 \\
\hline Event 7 & 221.1 & -6864 & -1595 & -1500 \\
\hline Event 8 & 22.4 & -5668 & -1622 & 0 \\
\hline Event 9 & 15.1 & -8527 & -2079 & 0 \\
\hline Event 10 & 224.1 & 5715 & 1418 & 0 \\
\hline Event 11 & 156.7 & 2087 & 730 & 3000 \\
\hline Event 12 & 211.8 & -14858 & -3780 & 4500 \\
\hline Event 13 & 290.8 & -3627 & -1052 & -1000 \\
\hline Event 14 & 119.4 & -80 & -173 & -1500 \\
\hline
\end{tabular}

Thus, this system parameter confirms the analyses of the delay and Doppler.

\section{CONCLUSION}

This paper reports the findings of anomalous artefacts in fourteen DDMs from real satellite TDS-1 data. It is found that the anomalous artefacts are not due to reflections from Earth surface targets, nor occultations, nor leakages of direct signals. The apparent delay of the anomalous artefacts suggests that they are reflections from targets above the Earth's surface, such as aircraft or spacecraft. It is also possible that other unknown reasons could lead to the anomalous artefacts. In this study, more than ten DDMs containing anomalous artefacts were found in the data over a period of less than two days. If the anomalous artefacts were due to reflections from targets above the Earth's surface, GNSS-R could be a feasible technique for detecting targets above the Earth's surface.

\section{REFERENCE}

[1]C. Hall and R. Cordey, "Multistatic scatterometry," in Geoscience and Remote Sensing Symposium, 1988. IGARSS'88. Remote Sensing: Moving Toward the 21st Century., International, 1988, pp. 561-562.

[2] M. P. Clarizia, C. Ruf, P. Cipollini, and C. Zuffada, "First spaceborne observation of sea surface height using GPS Reflectometry," Geophysical Research Letters, vol. 43, pp. 767774, 2016.

[3] G. Foti, C. Gommenginger, P. Jales, M. Unwin, A. Shaw, C. Robertson, et al., "Spaceborne GNSS reflectometry for ocean winds: First results from the UK TechDemoSat-1 mission," Geophysical Research Letters, vol. 42, pp. 5435-5441, 2015.

[4] A. Camps, H. Park, G. Portal, and L. Rossato, "Sensitivity of TDS-1 GNSS-R reflectivity to soil moisture: global and regional differences and impact of different spatial scales," Remote Sensing, vol. 10, p. 1856, 2018.

[5]A. Camps, H. Park, M. Pablos, G. Foti, C. P. Gommenginger, P.-W. Liu, et al., "Sensitivity of GNSS-R spaceborne observations to soil moisture and vegetation," IEEE Journal of Selected Topics in Applied Earth Observations and Remote Sensing, vol. 9, pp. 4730-4742, 2016.

[6] K. Yu, Y. Li, and X. Chang, "Snow Depth Estimation Based on Combination of Pseudorange and Carrier Phase of GNSS Dual-Frequency Signals," IEEE Transactions on Geoscience and Remote Sensing, 2018.

[7] A. Alonso-Arroyo, V. U. Zavorotny, and A. Camps, "Sea ice detection using UK TDS-1 GNSS-R data," IEEE Transactions on Geoscience and Remote Sensing, vol. 55, pp. 4989-5001, 2017.

[8] A. Di Simone, H. Park, D. Riccio, and A. Camps, "Sea Target Detection Using Spaceborne GNSS-R Delay-Doppler Maps: Theory and Experimental Proof of Concept Using TDS-1 Data," IEEE Journal of Selected Topics in Applied Earth Observations and Remote Sensing, vol. 10, pp. 4237-4255, 2017. 
[9]E. Valencia, A. Camps, N. Rodriguez-Alvarez, H. Park, and I. Ramos-Perez, "Using GNSS-R imaging of the ocean surface for oil slick detection," IEEE Journal of Selected Topics in Applied Earth Observations and Remote Sensing, vol. 6, pp. 217-223, 2013.

[10] A. Camps, H. Park, G. Foti, and C. Gommenginger, "Ionospheric effects in GNSS-reflectometry from space," IEEE journal of selected topics in applied earth observations and remote sensing, vol. 9, pp. 5851-5861, 2016.

[11] M. Unwin, P. Jales, J. Tye, C. Gommenginger, G. Foti, and J. Rosello, "Spaceborne GNSS-reflectometry on TechDemoSat-1: Early mission operations and exploitation," IEEE Journal of Selected Topics in Applied Earth Observations and Remote Sensing, vol. 9, pp. 4525-4539, 2016.

[12] C. S. Ruf, S. Gleason, Z. Jelenak, S. Katzberg, A. Ridley, R. Rose, et al., "The CYGNSS nanosatellite constellation hurricane mission," in Geoscience and Remote Sensing Symposium (IGARSS), 2012 IEEE International, 2012, pp. 214216.

[13] H. Carreno-Luengo, A. Camps, I. Perez-Ramos, G. Forte, R. Onrubia, and R. Díez, "3Cat-2: AP (Y) and C/A GNSS-R experimental nano-satellite mission," in 2013 IEEE International Geoscience and Remote Sensing Symposium-IGARSS, 2013, pp. 843-846.

[14] M. Martín-Neira, W. Li, A. Andrés-Beivide, and X. Ballesteros-Sels, "“Cookie": A Satellite Concept for GNSS
Remote Sensing Constellations," IEEE Journal of Selected Topics in Applied Earth Observations and Remote Sensing, vol. 9, pp. 4593-4610, 2016.

[15] J. Wickert, E. Cardellach, M. Martín-Neira, J. Bandeiras, L. Bertino, O. B. Andersen, et al., "GEROS-ISS: GNSS reflectometry, radio occultation, and scatterometry onboard the international space station," IEEE Journal of selected topics in applied Earth observations and Remote Sensing, vol. 9, pp. 45524581, 2016.

[16] P. Høeg, H. Fragner, A. Dielacher, F. Zangerl, O. Koudelka, P. Beck, et al., "PRETTY: Grazing altimetry measurements based on the interferometric method," in 5th Workshop on Advanced RF Sensors and Remote Sensing Instruments, ARSI'17, 2017.

[17] E. Cardellach, J. Wickert, R. Baggen, J. Benito, A. Camps, N. Catarino, et al., "GNSS Transpolar Earth Reflectometry exploriNg System (G-TERN): Mission Concept," Ieee Access, vol. 6, pp. 13980-14018, 2018.

[18] (2017). SMALLSATS WIN BIG PRIZE AT COPERNICUS MASTERS

Available:

https://www.esa.int/Our_Activities/Observing the_Earth/Copern icus/Smallsats_win_big_prize_at_Copernicus_Masters

[19] Manual. (2018). www.merrbys.org Mission and Product Descriptions. Available: http://merrbys.co.uk/wpcontent/uploads/2018/02/MERRByS-Product-Manual-V4.pdf 